\title{
NEUROMODULACIÓN DE RAÍCES SACRAS. EXPERIENCIA DE NUESTRO CENTRO 1998-2003. A PROPÓSITO DE 18 IMPLANTES DEFINITIVOS DE NEUROMODULACIÓN
}

\author{
I. GÓMEZ GARCÍA, E. FERNÁNDEZ FERNÁNDEZ*, E. SANZ MAYAYO, \\ S. CONDE SOMESO**, R. GARCÍA NAVAS, C. GUICIOS DORADO, A. LINARES QUEVEDO, \\ L. PERALES CABANAS, M. JIMÉNEZ CIDRE*, A. ESCUDERO BARRILERO
}

Unidad de Urodinámica. Servicio de Urología. Hospital Ramón y Cajal. *Integrantes del grupo español de Neuromodulación de Raices Sacras. **Servicio de Cirugía General. Hospital Ramón y Cajal.

Actas Urol Esp. 28 (10): 732-742, 2004

\section{RESUMEN}

NEUROMODULACIÓN DE RAÍCES SACRAS. EXPERIENCIA DE NUESTRO CENTRO 1998-2003. A PROPÓSITO DE 18 IMPLANTES DEFINITIVOS DE NEUROMODULACIÓN

OBJETIVO: La neuromodulacion de raíces sacras es una técnica eficaz para el tratamiento de la Disfunción Miccional Crónica (DMC), refractaria a tratamientos convencionales. En la actualidad están surgiendo nuevas indicaciones en el ámbito urológico, como el dolor pélvico crónico y la cistitis intersticial. Diversos grupos de cirujanos digestivos están optando por esta técnica para el tratamiento de patología rectal (estreñimiento, disfunción esfinteriana). Este trabajo aporta nuestra experiencia en el tratamiento de pacientes con DMC y los resultados comparativos al año del implante de todos los pacientes.

MATERIAL Y MÉTODOS: Desde diciembre de 1998 a julio del 2003 hemos realizados 18 implantes definitivos de neuromodulacion. La indicación principal fue la DMC, en un 62,5\% de los pacientes. El seguimiento de los pacientes lo realizamos al mes, tres meses, y posteriormente cada seis meses. Este se realiza mediante diario miccional y cuestionario de calidad de vida. El implante del electrodo en los 16 primeros pacientes se ha realizado mediante cirugia abierta, mientras que los 2 siguientes se ha colocado el electrodo mediante técnica percutánea, utilizando el kit "Tined Lead".

RESULTADOS: Comparamos resultados al año del implante en todos los pacientes. Se han realizado 18 implantes de neuromodulación de los cuales $14(77,7 \%)$ han sido en mujeres y $4(22,3 \%)$ en varones. La edad media es de 52,56 años. La indicación del implante en un $72,2 \%$ de los pacientes fue por DMC, un $22,1 \%$ por incontinencia mixta (urinaria y fecal), y un $5,5 \%$ fue por cistopatía intersticial. La mejoría de los síntomas al año del implante, medidos por el calendario miccional y el cuestionario de calidad de vida fue de $76,4 \%$. La mejoría clínica fue mayor en los pacientes con síntomas de urgencia, que los pacientes con predominio de la sintomatología de vaciado.

PALABRAS CLAVE: Neuromodulación. Raíces sacras. Disfunción miccional crónica. Electroestimulación.

\section{ABSTRACT \\ SACRAL ROOT NEUROMODULATION. EXPERIENCE IN OUR SITE: 1998-2003, CONCERNING 18 DEFINITE NEUROMODULATION IMPLANTS}

OBJECTIVE: Sacral root neuromodulation is an effective technique for the treatment of Chronic Micturition Dysfunction (CMD) refractory to conventional therapy. New indications such as chronic pelvic pain and interstitial cystitis are currently making their way within the urologic setting. Several groups of gastric surgeons are now choosing this technique for the management of rectal diseases (constipation, sphincter dysfunction). This paper contributes our experience in the treatment of patients with CMD and the comparative results at one year from implant in all patients.

MATERIAL AND METHODS: From December 1998 through July 2003, 18 neuromodulation definite implants were performed. The main indication was CMD in $62.5 \%$ patients. Follow up of patients was done at one month and three months, and every six months thereafter. Follow up is conducted through a micturition diary and QoL questionnaire. Electrode implant in the first 16 patients was achieved by open surgery. The next 2 patients had the electrode placed by a percutaneous technique using the "Tined Lead" kit.

RESULTS: Results at one year after implant were compared in all patients. The total number of neuromodulation implants placed was $18,14(77.7 \%)$ of which were women and $4(22.3 \%)$ men. Mean age was 52.56 years. Implant indication was CMD in $72.2 \%$ patients, mixed incontinence (urinary and faecal) in $22.1 \%$, and interstitial cystic disease in $5.5 \%$. Symptoms improvement at one year from implant, as determined by a micturition diary and GoL questionnaire was $76.4 \%$. Clinical improvement was greater in patients with urgency symptoms than in patients with predominance of voiding symptoms.

KEY WORDS: Neuromodulation. Sacral roots. Chronic micturition dysfunction. Electrostimulation. 
$\mathrm{L}^{2}$ a Neuromodulación de raíces sacras (NMRS), progresivamente se va imponiendo como técnica de elección en los pacientes con disfunción miccional crónica (DMC) refractaria a tratamiento farmacológico convencional. La principal indicación de esta técnica es la DMC, aunque paulatinamente se va abriendo el abanico de indicaciones terapéuticas como, el dolor pélvico crónico, la cistopatía intersticial, el estreñimiento, la incontinencia fecal. El correcto diagnóstico de la enfermedad, una adecuada orientación terapéutica, y aún más importante una correcta indicación de esta técnica; hacen de la NMRS una técnica de elección y efectiva en pacientes con DMC.

\section{MATERIAL Y METODOS}

Desde diciembre de 1998 a julio del 2003 se han implantado 18 neuromoduladores modelo Interstim de la casa Medtronic. Comparamos resultados al año del implante en todos los pacientes.

Desde agosto del 2003 hasta la actualidad, abril del 2004, se han implantado 8 neuromoduladores definitivos que no han sido incluidos en la serie por no haber llegado al año de seguimiento.

El estudio previo al implante conlleva una historia clínica general y urológica exhaustiva, solicitando a los pacientes candidatos para el implante: estudio urodinámico, estudio neurológico, cultivo de orina, citología, estudios ecográfico y urográfico. También proporcionamos al paciente un calendario miccional y cuestionario de calidad de vida (versión modificada de King's Health Questionnaire de 1993) que aportan datos objetivos sobre la calidad de vida en relación con la clínica miccional del paciente.

La principal indicación para el implante de neuromodulación de raíces sacras en nuestra serie ha sido la DMC en un 62,5\% de los casos; también se han realizando implante en pacientes con incontinencia mixta (urinaria y fecal), cistopatía intersticial y con Síndrome de Fowler.

Las contraindicaciones para la neuromodulación no han variado desde el estudio de la serie de Linares et $\mathrm{al}^{1}$ : gestación, infección aguda o crónica del tracto urinario, enfermedad oncológica, presencia de enfermedad neurológica, alteraciones de la anatomía o funcionalidad de la región sacra, incontinencia urinaria de esfuerzo, enfermedades sistémicas con repercusión neurológica, alteraciones mentales (psiquiátricas o intelectuales) que no capaciten al paciente para un correcto manejo del estimulador.

Previa la realización del implante, realizamos test de estimulación de nervios periféricos, para verificar la integridad del arco reflejo sacro, manteniendo posteriormente el electrodo de manera temporal no más de una semana, para evaluar la respuesta clínica a la estimulación. Si existe mejoría de los síntomas superior al $50 \%$ procedemos al implante del neuroestimulador.

El seguimiento de los pacientes lo realizamos al mes, tres meses, y posteriormente cada seis meses. Este se realiza mediante diario miccional y cuestionario de calidad de vida.

El implante del electrodo en los 16 primeros pacientes se ha realizado mediante cirugía abierta, mientras que los 2 siguientes se ha colocado el electrodo mediante técnica percutánea, utilizando el kit "Tined Lead". Los 8 implantes que no hemos incluido en la serie también se han realizado mediante técnica percutánea. La técnica percutánea utilizando el Tinead Lead es la siguiente:

$1^{\circ}$ Localización de la raiz S3 (Fig. 1).

$2^{\text {o }}$ Punción de S3 con aguja de punción. (Figs. 2 y 3).

3ํㅡㄹ Estimulación eléctrica y comprobación de reflejo plantar flexor y contracción anal. (Figs. 4 y 5).

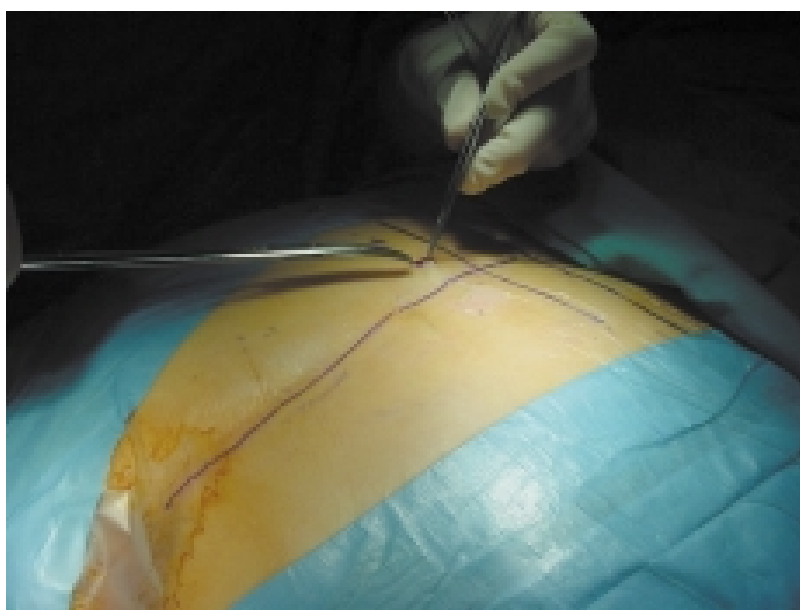

Figura 1 


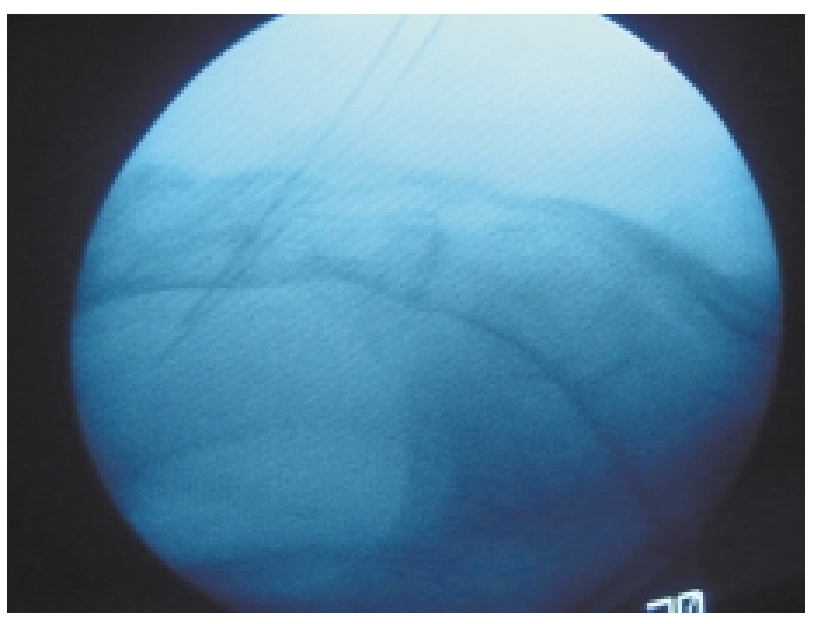

FIGURA 2

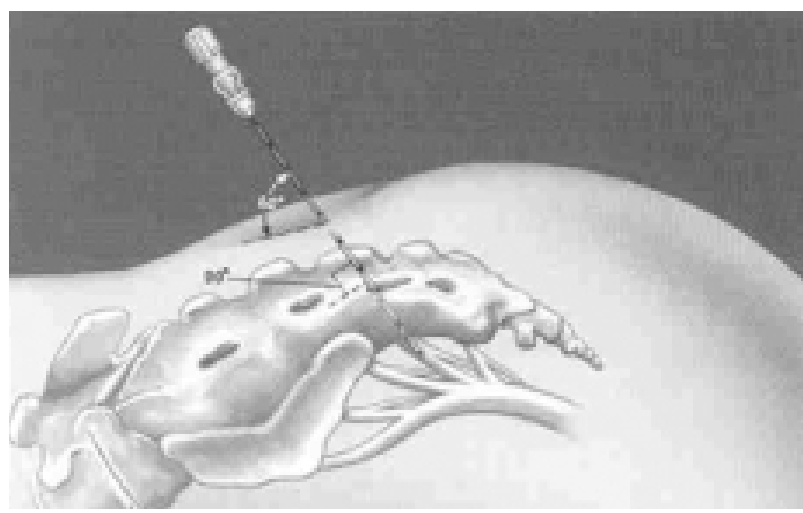

FIGURA 3

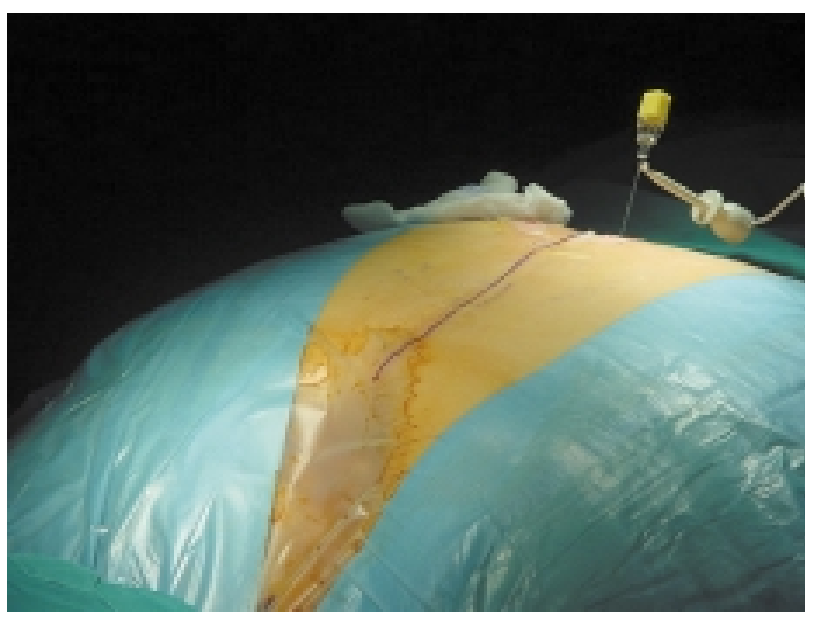

FIGURA 4

4ํ Paso de estilete a través de aguja de punción. (Figs. 6 y 7).

5을 Retirada de aguja de punción.

6을 Paso del kit introductor a través del estilete. (Figs. 8 y 9 ).

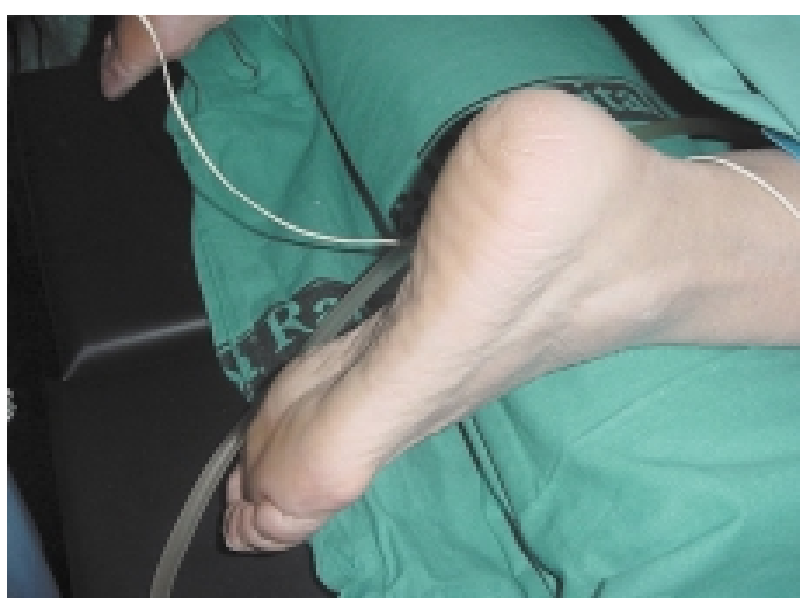

FIGURA 5

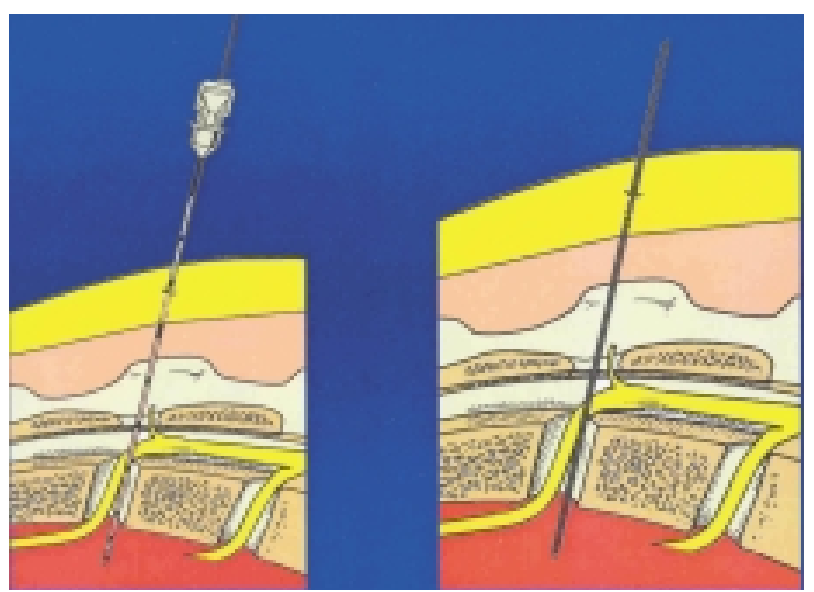

FIGURA 6

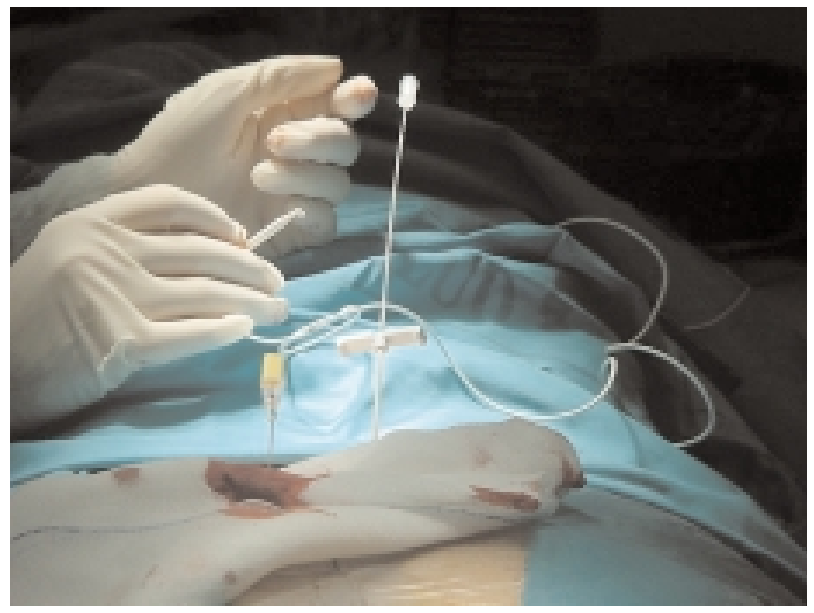

\section{FIGURA 7}

$7^{\text {o }}$ Retirada de estilete.

$8^{\text {o }}$ Introducción del electrodo por medio del kit introductor. (Figs. 10 y 11).

9ํㅡㄹ Retirada del kit introductor, y fijación del electrodo. 


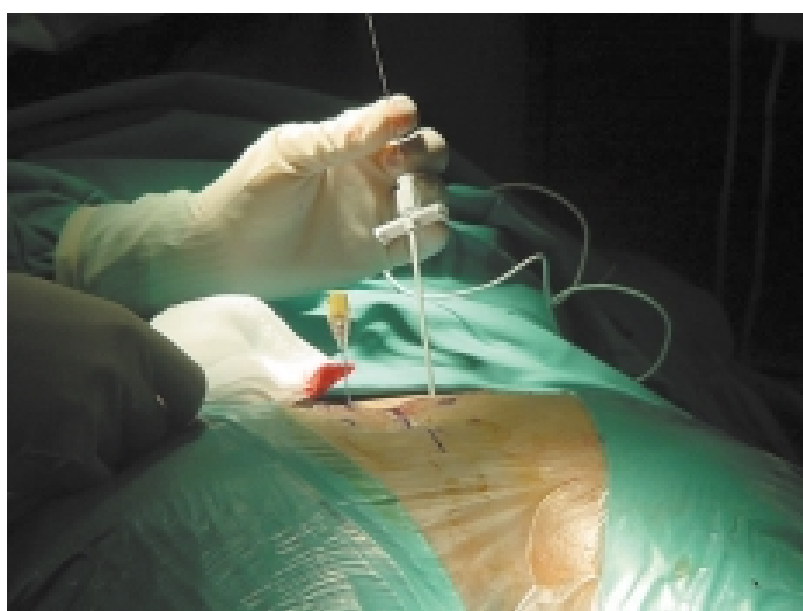

FIGURA 8

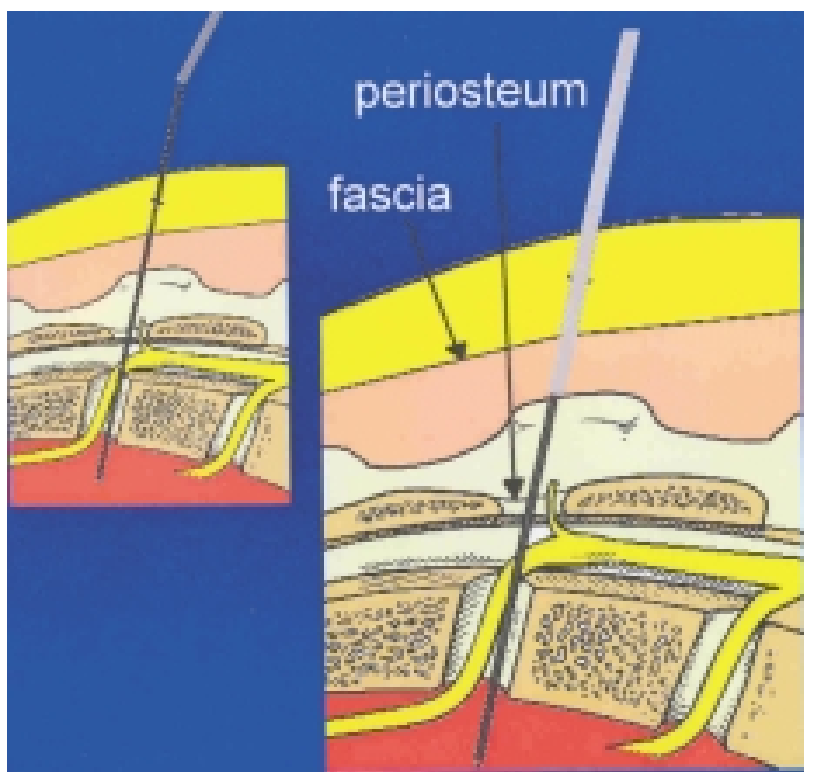

FIGURA 9

10ํㅡㄹ Comprobación de correcta localización del electrodo. (Figs. 12).

Si mejoría de los sintomas superior al 50\% a las tres semanas:

$11^{\circ}$ Implante de neuromodulador definitivo. (Figs. 13).

\section{RESULTADOS}

Los últimos datos del grupo español de neuroestimulación son de abril del 2003, habiéndose realizado hasta ese momento un total de 235 pacientes que han sido sometidos a una pruebas de neuroestimulación (PNE), siendo el 65\% mujeres (154), un 34\% hombres (79), no recogiéndose el sexo en dos de los pacientes. La edad media del

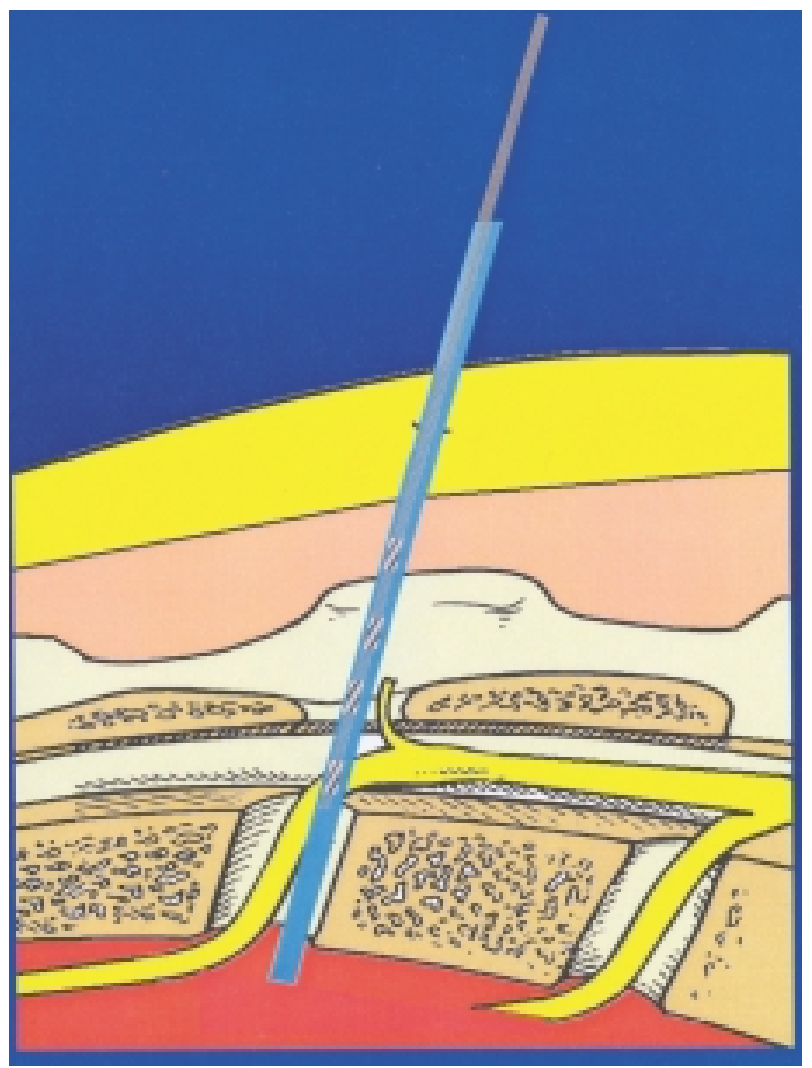

FIGURA 10

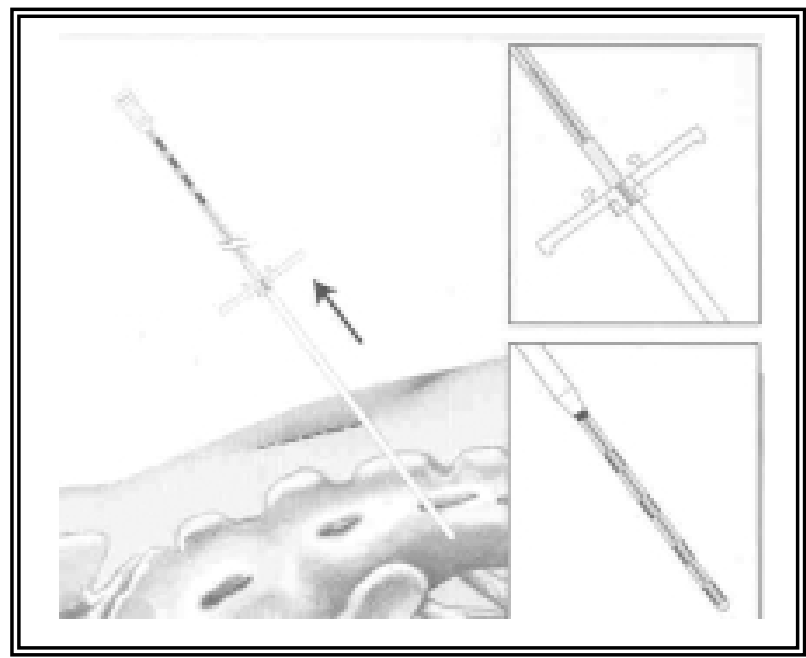

FIGURA 11

paciente testado fue de 47 años. De los 235 pacientes testados un $32 \%$ de los pacientes fueron implantados (76 pacientes), siendo 55 mujeres y 21 hombres. La sintomatología mas frecuente por la que se realizó el implante fue incontinencia por urgencia. 


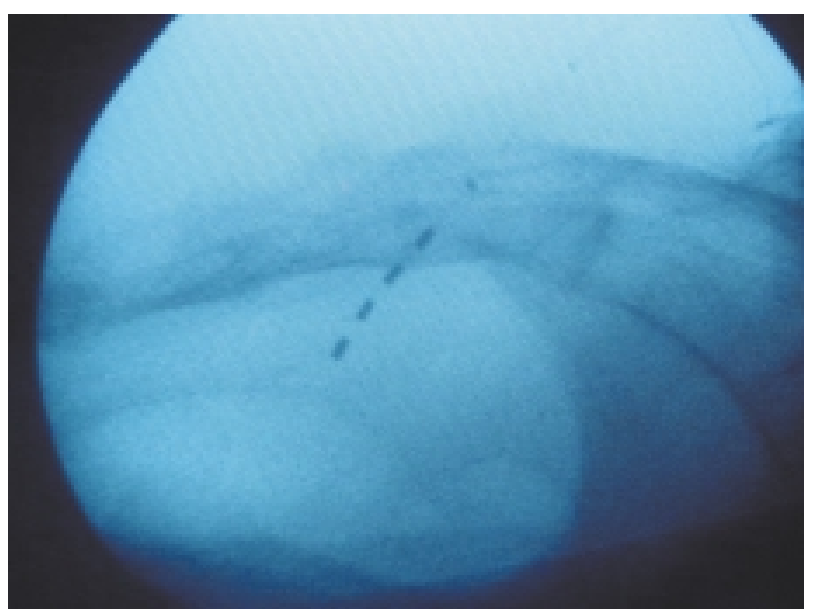

FIGURA 12

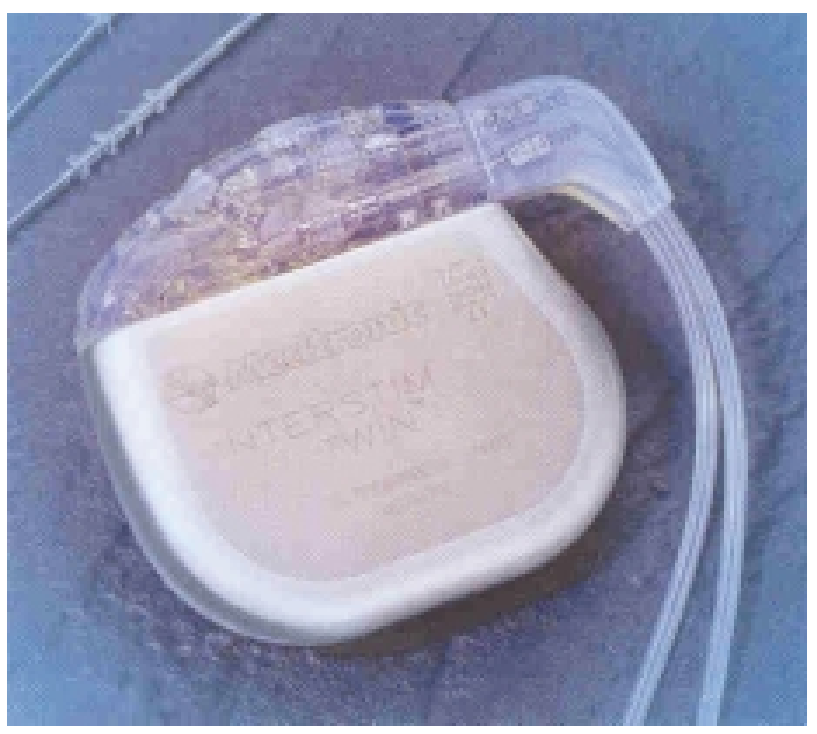

FIGURA 13

De los 76 pacientes implantados 18 han sido realizados en nuestro centro, los 10 primeros se implantaron entre diciembre de 1998 y enero del 2002 hasta julio del 2003. En este periodo se han realizado un total de 49 pruebas de neuroestimulación (PNE), y un total de 18 implantes lo que supone una tasa de implante del $36,73 \%$. Los pacientes incluidos en esta serie, presentado como mínimo un año de seguimiento; siendo los resultados aquí presentados al año del implante.

a) Serie de diciembre de 1998 a enero del 2002 (10 implantes):

Del primer estudio de nuestro hospital (19982001) el $80 \%$ eran mujeres y un $20 \%$ hombres, siendo la edad media de 49 años ( rango de 13-71 años). La indicación para el implante en 8 de ellos fue por disfunción miccional crónica (DMC): 2 de ellos por retención urinaria (20\%), en 3 trastornos miccionales mixtos (30\%), y en $3(30 \%)$ presentaban trastornos de frecuencia e incontinencia por urgencia. Los otros dos pacientes implantados fue por incontinencia mixta (fecal y urinaria $(20 \%))$.

b) Serie de enero del 2002 hasta julio del 2003 (8 implantes):

Entre enero del 2002 hasta julio del 2003 se han implantado 8 neuromoduladores definitivos de estimulación de raíces sacras. La media de edad fue de 57,4 años con un rango de 29 a 74 años. De estos ocho pacientes seis eran mujeres $\mathrm{y}$ dos hombres, lo que supone respectivamente el $75 \%$ y el $25 \%$. El $75 \%$ de los pacientes (6) habían recibido tratamiento con anticolinérgicos, un paciente $(12,5 \%)$ estaba con cateterismos intermitente, y un $(12,5 \%)$ paciente refería instilaciones previas con Dimetil sulfoxido (DMSO). Dos de las pacientes (25\%) habían sido sometidas a cirugía de incontinencia, mediante implante vaginal de malla de propileno (TVT), y un varón $(12,5 \%)$ se había sometido a una cérvico-prostatotomía. (Gráfico 1).

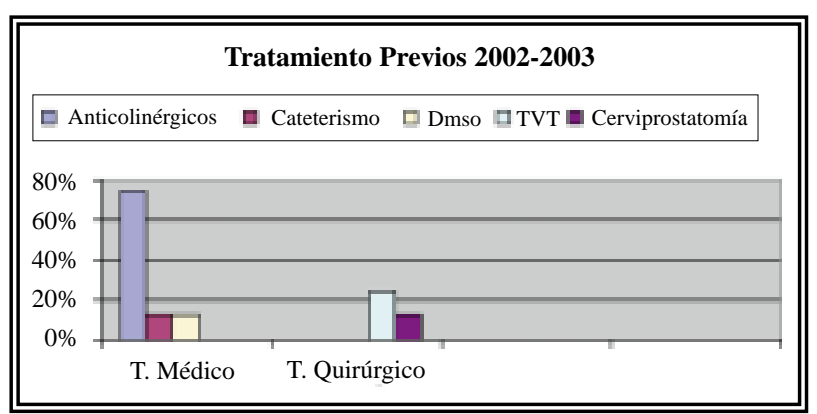

GRÁFICO 1

Respecto a la etiología de su disfunción miccional existían tres pacientes con disfunción miccional crónica $(37,5 \%)$, tres pacientes con vejiga neurógena $(37,5 \%)$, un paciente con cistopatía crónica $(12,5 \%)$, otro paciente con síndrome de Fowler $(12,5 \%)$. De estos ocho pacientes dos (25\%) presentaban alteraciones en la continencia fecal asociadas al trastorno miccional.

Tras la realización de la prueba de neuroestimulación ambulante, se procedió a colocar bajo 
anestesia general el neuromodulador definitivo, colocándose en el 100\% de los casos a nivel subcutáneo derecho, con el electrodo estimulando el haz anterior de la raiz derecha de S3. De los 8 implantes 6 se realizaron con cirugía abierta y dos mediante técnica percutánea. Las revisiones se realizaron en los pacientes implantados al mes, tres meses y año. La mejoría de la clínica miccional fue del $76,4 \%$ de media, con respecto a la situación clínica previa al implante. En los dos pacientes en que se les realizó un implante por incontinencia mixta (urinaria y fecal), la mejoría de la clínica miccional fue del $92,5 \%$, mientras que la mejoría de la clínica de la incontinencia fecal fue entorno al $70 \%$.

En cuanto a los efectos secundarios: un paciente $(12,5 \%)$ presento dolor sacro en el área del implante, que cedió con toma de analgésicos; y dos (25\%) de ellos refirieron sensaciones parestésicas en miembro inferior derecho, que desaparecieron con la reprogramación del neuromodulador. Una $(12,5 \%)$ de nuestras pacientes presentó reagudización de cuadros pseudoclusivos digestivos, previamente conocidos, no pudiendo demostrar relación causa efecto entre el implante del neuromodulador y la reagudización de la clínica digestiva. En una paciente fue necesario la retirada del neuromodulador por infección.

c)Resultados globales de nuestro centro; diciembre de 1998 a julio del 2003 (18 implantes):

Los resultados globales de los 18 implantes realizados en el Hospital Ramón y Cajal, entre diciembre de 1998 y julio del 2003, son los siguientes:

Se han realizado 18 implantes de neuromodulación de los cuales $14(77,7 \%)$ han sido en mujeres y $4(22,3 \%)$ en varones. La edad media es de 52,56 años con un rango de 13 a 74 años. La indicación del implante en un $72,2 \%$ (13) de los pacientes fue por DMC, en un $22,1 \%$ (4) por incontinencia mixta (urinaria y fecal), en un 5,5\% (1) fue por cistopatía intersticial. La mejoría de los síntomas al año del implante, medidos por el calendario miccional y el cuestionario de calidad de vida fue de $76,4 \%$. De los 13 pacientes con DMC, siete $(38,8 \%)$ presentaban cuadro de frecuencia con urgencia mejorando en estos su cuadro miccional en un $79,5 \%$ de media; los otros 6 pacientes con DMC presentaban predominio de los síntomas de vaciado, alcanzando una mejoría de los síntomas entorno 72,3\% (Gráfico 2).

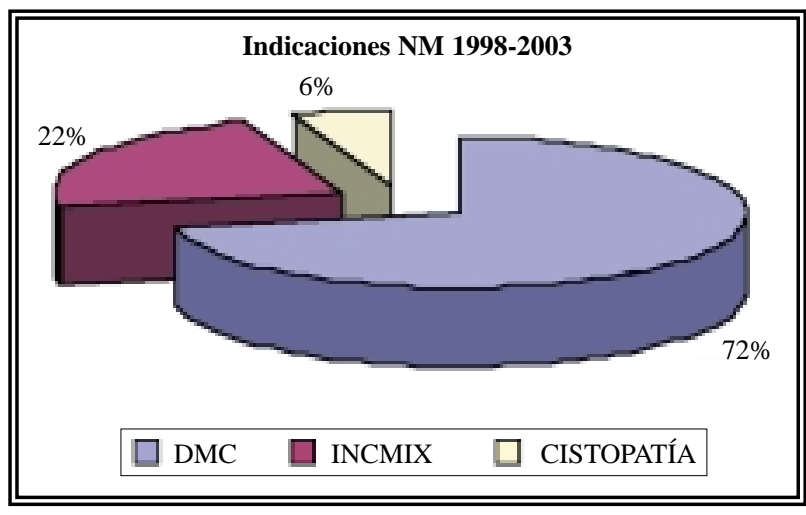

GRÁFICO 2

Los cuatro pacientes que presentaban incontinencia mixta obtuvieron una mejoría de la frecuencia del 92,5\% de media, siendo la mejoría de la clínica fecal entre el 65 al 70\%. La paciente con cistopatía intersticial presentó una mejoría de su sintomatología del 73\% (Gráfico 3).

De los 18 implantes, 16 se realizaron con cirugía abierta y dos mediante técnica percutánea. En los 18 implantes, hemos tenido una complicación mayor que llevó a la extracción del implante, con complicaciones menores en el $27,7 \%$ (5 pacientes), como parestesias en miembro inferior ipsilateral, dolor mecánico en la zona del implante, y seroma postquirúrgico (Gráfico 4).

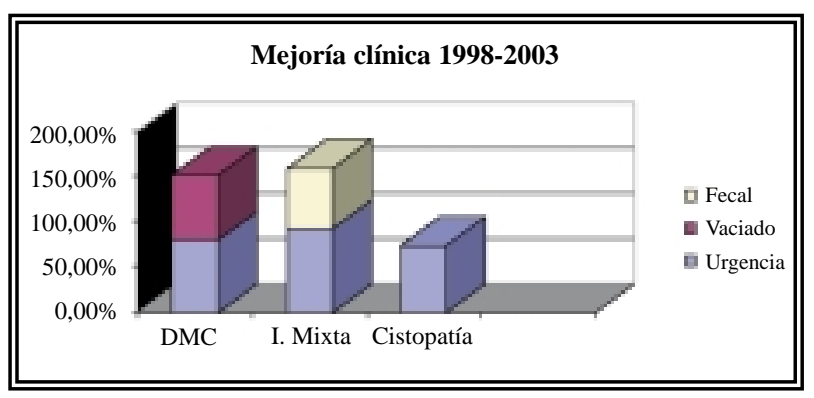

GRÁFICO 3

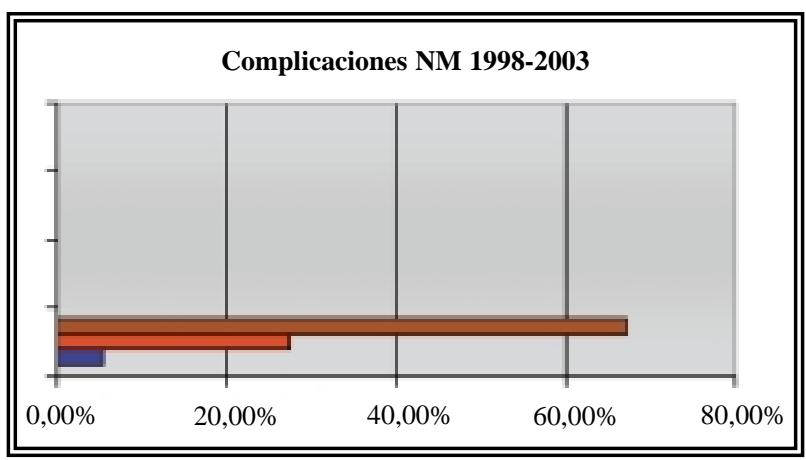

GRÁFICO 4 


\section{DISCUSIÓN}

La neuromodulación (NM) de raíces sacras es un tratamiento efectivo y resolutivo en pacientes con disfunción miccional crónica (DMC), refractaria al tratamiento convencional. La DMC es la principal indicación para la NM, siendo otras indicaciones la cistitis intersticial, dolor pélvico crónico, incontinencia fecal, estreñimiento...

En los 20 últimos años ha existido un progresivo cambio, en el enfoque terapéutico de la disfunción del tracto urinario inferior, abandonándose técnicas como la ampliación vesical y surgiendo nuevas como la electroestimulación. De manera genérica el mecanismo de actuación de la estimulación eléctrica de raíces sacras es por su capacidad de estabilizar, modular, reestablecer los reflejos miccionales alterados.

La electroestimulación consiste en aplicar impulsos eléctricos para modificar el comportamiento de un arco neuronal deficiente. La electroestimulación se divide en central y periférica. La central o neuroestimulación, estimula las vías eferentes nerviosas para crear una respuesta motora, siendo utilizada en pacientes con lesiones medulares. La electroestimulación periférica o neuromodulación crea un campo eléctrico que estimula las raíces aferentes, consiguiendo modificar el comportamiento del detrusor, esfinter uretral externo, y músculos del suelo pélvico. El mecanismo de acción de la neuromodulación se basa en la estimulación de las fibras aferentes somáticos, modulado los reflejos miccionales, y vías nerviosas en médula espinal.

El centro de la micción a nivel medular se encuentra entre los segmentos S2-S4, siendo S3 el sitio de mas habitual de localización. En la sustancia gris pontomesencefálica se encuentran los núcleos de origen para la vía común de las neuronas motoras de la vejiga; recibiendo estos centros impulsos nerviosos superiores. La micción se consigue por una correcta coordinación entre la actividad del detrusor y los esfinteres, tanto vesical como el estriado.

En el siglo dieciocho Luigi Galvani demostró en sus experimentos con ranas la capacidad contráctil del músculo al aplicarse electricidad, sirvieron para asentar las bases que en el futuro, servirían para elaborar las teorías sobre el potencial transmembrana y la conducción eléctrica nerviosa. Posteriormente en 1800 los estudios de Alessandro Volta demostraron que la contracción muscular es debida a la estimulación nerviosa. En 1820 los ensayos realizados por Oersted y Faraday sobre la relación existente entre el magnetismo y la electricidad, sirven en la actualidad como base de la neuromodulación. Saxtorph en 1876, realiza la primera estimulación transuretral en un paciente con retención aguda de orina.

En 1960 Mcguire y posteriormente Boyce, realizaron modelos experimentales de electroestimulación sobre perros, demostrando la importancia del tamaño del electrodo, y que la estimulación a ambos lados de la vejiga producían la máxima respuesta. En 1963 Bradley et al. realizan electroestimulación sobre distintos animales, y observo que en vejigas grandes se necesita mayor potencia y mayor área de contacto entre los electrodos y la zona a estimular.

Nashold y Friedmanson, son los primeros que investigan sobre la estimulación nerviosa espinal para alteraciones miccionales, observando que el área optima de estimulación eléctrica son de S1 a S3. Jonas et al. observaron que la estimulación de los centros nerviosos produce contracción del músculo liso uretral, del detrusor y del esfínter estriado. Posteriormente Thürhoff determinó la existencia de dos núcleos, el parasimpático y el núcleo pudendo; siendo el primero el responsable de la contracción del detrusor, y el segundo del esfinter estriado; así la estimulación independiente del parasimpático produciría solo estimulación del detrusor sin estimular el esfinter estriado. En 1976 Brindley descubre que la tercera raiz sacra (S3) es la que mas influye en el control miccional. Tanhago et al. demuestra que no existe diferencia entre la estimulación del lado derecho o del izquierdo, también apreció que la estimulación de las raíces ventrales sacras es más eficaz. El estudio histológico de las fibras estimuladas no evidenció alteraciones respecto a las fibras contralaterales no estimuladas.

En España en 1980 los Drs. Tallada, Perales y Romero realizan un estudio en estimulación de raíces sacras en gatos investigando; que raiz estimulada produce mejor respuesta vesical, y que parámetros son los mas efectivos para la estimulación. En 1998 Bosch et al. demuestran que la estimulación crónica vesical, no disminuye el efecto regulador. 
En 1981 los profesores Tanagho y Schmidt de la universidad de California realizan el primer implante de neuromodulación en un paciente con trastornos de la micción. En agosto de 1997 la FDA americana aprueba la neuroestimulación como técnica valida para el tratamiento de la incontinencia por urgencia. En 2002 la FDA americana aprueba la utilización del Tined Lead. En España en 1998 el Dr. Tallada implanta el primer neuromodulador. Actualmente hay en el mundo más de 10.000 pacientes implantados.

La DMC es un grupo de alteraciones miccionales que se caracterizan por alteraciones en los sistemas facilitadores e inhibidores de la micción. La DMC abarca un grupo de enfermedades que se caracterizan por alteraciones en el llenado o vaciado vesical, manifestándose clínicamente por cuadros de incontinencia de urgencia, síndrome de urgencia frecuencia, alteraciones en el vaciado vesical, y dolor pélvico con la micción, sin causa orgánica o neurológica. La DMC es la indicación principal de la neuromodulación de raíces sacras. En la actualidad las indicaciones de la neuromodulación abarcan enfermedades como el dolor pélvico crónica, el síndrome de Fowler, la cistopatía intersticial, empezando progresivamente a emerger nuevas indicaciones para esta técnica.

Las principales indicaciones para la estimulación de los nervios sacros, son pacientes con disfunción miccional crónica refractaria al tratamiento convencional. Las indicaciones más usuales son $^{2}$ :

- Incontinencia urinaria de urgencia secundaria a vejiga neurógena.

- Síndrome urinario de frecuencia urgencia.

- Trastornos en el vaciado vesical.

- Retención urinaria de repetición sin objetivación de patología obstructiva.

- Dolor pélvico crónico.

- Vejiga neurógena.

- Cistitis intersticial.

- Trastornos fecales ( incontinencia, retención crónica).

a) Incontinencia urinaria urgencia por vejiga neurógena: Cuando se instaura una interrupción entre la neurona superior con la vejiga se produce hiperreflexia del detrusor. En los pacientes en que el tratamiento con anticolinérgicos, asociado o no a autosondajes, se ha demostrado la efectividad de la neuroestimulación mediante la estimulación de las fibras somáticas ${ }^{3,4}$, ya que produce una inhibición de la contractibilidad vesical, siendo S3 el haz nervioso más adecuado para la estimulación crónica. Diversos estudios han ofrecido óptimos resultados en pacientes con lesión medular y esclerosis múltiple. La eficacia de la neuromodulación en pacientes con vejiga hiperrefléxica secundaria a esclerosis múltiple o sección incompleta de la médula está alrededor del $50 \%$ según las series ${ }^{5,6}$; mientras que Hohenfellner et al. no encontraron ningún tipo de respuesta en pacientes con lesión completa de la médula espinal ${ }^{7}$.

b) Incontinencia urinaria de urgencia: Puede ser debida a vejiga hiperrefléxica o inestable, caracterizándose ambas por contracción involuntaria del detrusor, que conlleva a pérdida involuntaria de orina con sensación de urgencia. NRS ha demostrado ser efectivo en la incontinencia de urgencia refractaria al tratamiento convencional, considerándose como efectivo cuando existe reducción de más del $50 \%$ de los síntomas en un plazo entre 18-72 meses después del implante ${ }^{8}$. Estudios multicéntricos han demostrado una efectividad del tratamiento del 41 al $100 \%{ }^{9}$. La estimulación de las raíces sacras produce modulación de los reflejos sacros estabilizando la función del detrusor aumentando el tono y equilibrando la musculatura del suelo pélvico.

c) Sindrome de urgencia frecuencia: Es otra manifestación de hiperactividad de la vejiga. Se caracteriza por una imperiosa necesidad de orinar, con mínimas cantidades de orina vaciadas, y una frecuencia cada 15 minutos; ocasionalmente este cortejo se puede acompañar de dolor y de incontinencia, siendo este último un hecho secundario en la clínica. Urodinámicamente estos pacientes, no presentan hiperactividad del detrusor. La teoría de este síndrome es incierta, siendo una de las más aceptadas alteraciones del arco reflejo sacro. En el estudio de Hassouna et $\mathrm{al}^{10}$, se observó que con NRS era beneficioso en el $56 \%$; observando que aumenta la cantidad de la diuresis con cada vaciado, disminución de la 
frecuencia miccional. Otros estudios objetivaron beneficio del 43-83\% en el síndrome de urgencia frecuencia con la neuromodulación de raíces $\operatorname{sacras}^{9,11}$.

d) Dificultad miccional: Problemas de vaciado vesical pueden ser debidos por falta de contracción vesical, y/o elevada resistencia uretral. La etiología puede ser orgánica o funcional, pudiendo ser a la primera resuelta en la mayoría de los casos mediante cirugía. La etiología funcional es difícil de tratar ya que habitualmente se desconoce la causa. Fisiopatológicamente es un detrusor hipoactivo o existe una disinergia vesicoesfinteriana. La clínica varía desde retención urinaria completa o parcial, pasando por diversos trastornos miccionales de características obstructivas (debilidad de calibre miccional, estranguria...). Mediante la modulación se intenta conseguir estabilizar los reflejos miccionales logrando una micción coordinada.

e) Síndrome de Fowler: Descrito por primera vez en 1988 por Fowler, que se observa en mujeres jóvenes, manifestándose en un cuadro clínico caracterizado fundamentalmente por retención urinaria, secundario a un aumento de la contracción del esfinter uretral estriado. La electromiografía se aprecia contracción elevada del esfínter uretral, que se define acústicamente como "ruido de ballenas" que denota una actividad de tipo miotónico. Fowler también observó que las mujeres con este sindrome presentaban en su mayoría ovarios poliquísticos sugiriendo que la contracción anormal o ausencia y/o alteración de la relajación del esfinter podía estar mediada hormonalmente. Las pacientes con este sindrome son buenas candidatas para la neuromodulación ${ }^{12}$.

f) Cistitis intersticial: Es un diagnostico de exclusión siendo un cuadro que genéricamente se caracteriza por dolor pelviano crónico y síndrome miccional con intensa frecuencia y urgencia, sin identificarse su causa. La diversidad de etiologías en esta entidad hace que la mayoría de los tratamientos sean empíricos. El mecanismo de actuación de la neuromodulación sobre la cistitis intersticial se desconoce, aunque ha demostrado que disminuye los síntomas de urgencia y de frecuencia ${ }^{13}$. g) Dolor pélvico: El diagnóstico del síndrome de dolor pélvico crónico, es por exclusión de patología inflamatoria, irritativa, o tumoral. La incidencia en las mujeres en edad fértil es de hasta el 15\%, siendo la incidencia del hombre desconocida, ya que muchos de estos cuadros se catalogan de prostatitis abacteriana o prostatodinia ${ }^{14}$. La patogenia de este síndrome es desconocida, pero es habitual encontrar dos hechos comunes, la hiperactividad del suelo pélvico y la congestión pelviana. Usualmente están asociados a clínica diversa como la dificultad en el vaciado vesical, dispauremia, estreñimiento... La eficacia de la neuromodulación en pacientes con esta dolencia, se basa en el bloqueo de la transmisión del dolor en las vías espinotalámicas. Evidenciaron disminución del dolor en alrededor de un 50\% de los pacientes, no encontrándose a los 36 meses fracasos en el tratamiento.

h) Incontinencia fecal: La indicación inicial de esta técnica es sobre pacientes con incontinencia fecal, secundaria a disminución de la actividad del músculo estriado en la que han fracasado la cirugía; en la actualidad se están incluyendo otras indicaciones como lesiones parciales post quirúrgicas del esfinter, alteraciones esfinterianas post traumatismo medular, lesiones no completas de ambos esfinteres, lesiones del músculo liso del esfïnter interno ${ }^{15,16}$. El mecanismo de actuación es multifactorial, siendo dos de ellos: la elevación del ángulo colorrectal, y aumentando la presión de cierre del esfinter anal, mediante la estimulación del músculo estriado del suelo pélvico. Existen dos haces nervios que producen estimulación de la musculatura estriado del suelo pélvico, la cual favorece la continencia anal, siendo el sitio de estimulación mas distal de ellos las raíces sacras. Durante la prueba de estimulación se observa la respuesta del esfinter anal a la estimulación selectiva de cada raiz sacra, siendo la prueba positiva cuando se observa movimiento del esfínter anal, del suelo pélvico o se observa respuesta en la electromiografía o en la manometría anal $^{17}$. Si los síntomas disminuyen con la prueba de estimulación, o el paciente recurre su sintomatología al retirarlo, se considera al paciente como buen candidato para implante definitivo. La mejoría sintomática con la neuromodulación varía de un $85 \%$-100\% según las series ${ }^{15,18}$. 
i) Estreñimiento: Existen diversas causas de estreñimiento que pueden ser corregidas mediante cirugía (rectocele, prolapso rectal...), pero la disfunción o la pérdida de coordinación entre el recto y la tonicidad del esfinter anal puede ser causa de estreñimiento crónico. Este último caso puede ser corregido mediante estimulación de raíces sacras. En el estudio realizado por Agachan et $\mathrm{al}^{19}$, demostraron una disminución del índice de Wexner, disminución de la dificultad de vaciado rectal, disminución del tiempo en la evacuación y disminución en la dificultad de la evacuación; concluyendo este estudio que los pacientes con estreñimiento crónico secundario a disfunción esfíntero rectal se pueden beneficiar de la estimulación de raíces sacras, aunque todavía queda camino por recorrer. En la actualidad se estudian otras indicaciones de la neuromodulación rectal como en el síndrome de úlcera rectal solitaria, y el estreñimiento del colon inertia.

Las complicaciones de esta técnica varían según las series del 22 al $43 \%^{20,21}$, con una incidencia de reintervención variable según las series del 6 al $50 \%{ }^{22,23}$.

El estudio The Medtronic (MDT-103), multicéntrico en Norteamérica y Europa englobó a 633 pacientes siendo implantados en 250 de ellos un NRS. En 157 (62\%) de los pacientes implantados se detectó un total de 368 efectos adversos. Del total de los efectos adversos un 15,2\% no requirieron actitud alguna, a un $41 \%$ se les realizó terapias no quirúrgicas, y en el $43 \%$ de los pacientes fue necesaria la reintervención quirúrgica; siendo el problema resuelto en el 89,4\% de los casos. Los efectos adversos mas habituales son los que se nombran en la Tabla 1 .

Tabla 1

\begin{tabular}{lcc}
\hline Efecto adverso n/\% & MDT-103 & $\begin{array}{c}\text { Serie } \\
\text { Ramón y Cajal }\end{array}$ \\
\hline Dolor en implante & $60(14,2 \%)$ & $1(5,5 \%)$ \\
Otros dolores & $50(10,8 \%)$ & $3(16,5 \%)$ \\
Infección & $28(7 \%)$ & $1(5,5 \%)$ \\
Dolor en trayecto electrodo & $18(5,5 \%)$ & \\
Alteraciones intestinales & $12(3 \%)$ & $1(5,5 \%)$ \\
Migración del electrodo & $39(9,1 \%)$ & \\
Pérdida de eficacia & $13(3,53 \%)$ & \\
\hline
\end{tabular}

De los pacientes que necesitaron reintervención quirúrgica la causa más frecuente fue por alteraciones en la función o situación del electrodo o extensión, debido a emigración del electrodo, alteraciones sensitivas o motoras de las piernas, pérdida de eficacia o dolor en la zona del implante; siendo solucionado en la mayor parte de los casos reimplantando el aparato con anestesia local. En otros casos fue necesario extraer temporalmente el neuromodulador siendo ocasionado por diversas causas entre las que hay que reseñar infección, dolor pélvico crónico, alteraciones sensitivas rectales. También fue necesario cambio del electrodo sobre todo por pérdida de eficacia, descargas eléctricas y problemas técnicos del aparato.

\section{CONCLUSIONES}

La neuromodulación de las raíces sacras es una técnica eficaz para el tratamiento de la DMC, en pacientes en los que no existe mejoría o curación con los tratamientos convencionales. La neuromodulación en nuestra serie ha sido más efectiva en pacientes con sintomatología de urgencia/frecuencia (mejoría alrededor del 80\%) que pacientes con alteraciones del vaciado vesical (mejoría del 72\%).

Desde el primer implante en 1981 por el profesor Tanagho las indicaciones van siendo más amplias, incluyendo dolor pélvico crónico, incontinencia fecal, cistitis intersticial. En la actualidad existen estudios controvertidos sobre la aplicación de la neuromodulación en trastornos miccionales secundarios a enfermedades neurológicas como la esclerosis múltiple, parkinson; y lesiones medulares parciales, lo que abre un futuro prometedor para la aplicación de esta técnica en múltiples patologías que hasta el momento actual no tenía un tratamiento efectivo ${ }^{24}$.

\section{REFERENCIAS}

1. Linares Quevedo AI, Jiménez Cidre MA, Fernández Fernández E. et al. Nuromodulación de raíces Sacras posteriores en el tratamiento de la disfunción miccional crónica. Actas Urol Esp 2002;26:250-260.

2. Scheepens WA, Van Kerrebroeck PEV. Indications and predictive factors. Cap 9. In: New perspectives in sacral nerve stimulation. Ed. Martin Dunitz UK. 2002.

3. Hassouna MM, Siegel SW, Nyeholt AA, Elhilali MM. Sacral neuromodulation in the treatment of urgency-frequency symptoms: a multicenter study on efficacy and safety. $\mathrm{J}$ Urol. 2000 jun;163(6):1849-1854. 
4. Walsh IK, Johnston RS.Transcutaneous sacral neurostimulation for irritative voiding dysfunction. Eur Urol. 1999; 35(3): 192-196.

5. Ishigooka M, Suzuki Y, Hashimoto T. A new technique for sacral nerve stimulation: a percutaneous method for urinary incontinence caused by spinal cord injury. Br J Urol 1998 feb;81(2):315-318.

6. Chartier-Kastler EJ, Ruud Bosch JL, Perrigot M. Longterm results of sacral nerve stimulation (S3) for the treatment of neurogenic refractory urge incontinence related to detrusor hyperreflexia. J Urol 2000 nov;164(5):1476-1480.

7. Hohenfellner M, Dahms SE, Matzel K. Sacral neuromodulation for treatment of lower urinary tract dysfunction. BJU Int 2000 may;85 Suppl 3:10-19; discussion 22-23. Review.

8. Weil EH, Ruiz-Cerdá JL, Eerdmans PH. Sacral root neuromodulation in the treatment of refractory urinary urge incontinence: a prospective randomized clinical trial. Eur Urol 2000 feb;37(2):161-171.

9. Weil EH, Ruiz-Cerdá JL, Eerdmans PH. Clinical results of sacral neuromodulation for chronic voiding dysfunction using unilateral sacral foramen electrodes. World J Urol 1998;16(5):313-321.

10. Hassouna MM, Group NSS. Effect of sacral neuromodulation on urinary urgency frecuency. J. Urol 1999, 162: 254

11. Shaker H, Hassouna MM. Sacral root neuromodulation in the treatment of various voiding and storage problems. Int Urogynecol J Pelvic Floor Dysfunct 1999;10(5):336-343. Review.

12. Swinn MJ, Kitchen ND, Goodwin RJ. Sacral neuromodulation for women with Fowler's syndrome. Eur Urol 2000 oct;38(4):439-443.

13. Chai TC, Zhang C, Warren JW:Percutaneous sacral third nerve root neurostimulation improves symptoms and normalizes urinary HB-EGF levels and antiproliferative activity in patients with interstitial cystitis. Urology 2000 may;55(5):643-646.

14. Stones RW, Selfe SA, Fransman S, et al. Phsychosocial and economic impact oc chronic pelvic pain. Baillieres best pract res clinobstet Gynaecol 2000;14:415-431.

15. Matzel KE, Stradelmaeier U, Hohenfellner M. et al. Electrical stimulation of sacral spinal nerve for treatment of faecal incontinence. Lancet 1995;346;1124-1127.
16. Matzel KE, Stradelmaeier U, Hohenfellner M. et al. Cronical sacral nerve stimulation for faecal incontinence : long term results with foramen and cuff electrode. Dis colon Rectum;44:59-66.

17. Vaizey CJ, Kamm MA, Turner IC, et al. Effect of short term sacral nerve stimulationon anal and rectal function in patients with anal incontinence. Gut 1999;44:407-412.

18. Malouf AJ, Vaizey CJ, Nicholls RJ, et al. Permanent sacral nerve stimulation for faecal incontinence. Ann Surg 2000;232:143-148.

19. Agachan F, Chen T, Pfeifer J, et al. A constipation score system to simplify evaluation and management of constipated patients. Dis Colon Rectum 1996;39:681-685.

20. Dijkema HE, Weil EH, Mijs PT, et al. Neuromodulation of sacral nerves for incontinence and voiding dysfunctions. Clinical results and complications. Eur Urol 1993;24:7276.

21. Grünewald V, Höfner K, Thon WF et al. Sacral electrical neuromodulation as an alternative treatment option for lower urinary tract. Restol Neurol Neurosci 1999;14:189193.

22. Bosch R, Groen J. Sacral segmental nerve stimulation as a treatment for urge incontinence in patiens with detrusor instability: results of chronic electrical stimulation using and implantable neural prsthesis. J Urol 1995;154:504507.

23. Koldewijn EL, Meuleman EJ, Bemelmans BLH, et al. Neuromodulation effective in voiding dysfunction despite high reoperation rate. $J$ Urol. 255 (abstract 984).

24. Ruiz Cerdá JL, Martínez Agulló E, Jiménez Cruz F, Arlandis Guzmán S. Neuromodulación, una nueva alternativa terapéutica para los trastornos del tracto urinario inferior. Tema Monográfico LXV Congreso Nacional de Urología. Mayo 2000.

Dr. I. Gómez García

María Tubau, 15 - portal 3, $1^{\circ} 3$

28050 Madrid

(Trabajo recibido el 15 abril de 2004) 